\title{
Unsteady Flow Characteristics in a 90 Degree Elbow Affected by Developed, Undeveloped and Swirling Inflow Conditions*
}

\author{
Yukiharu IWAMOTO**, Manabu KONDO***, Hirotaka MINAMIURA ${ }^{\dagger}$, \\ Masaaki TANAKA ${ }^{\dagger \dagger}$ and Hidemasa YAMANO ${ }^{\dagger \dagger}$ \\ ** Graduate School of Science and Engineering, Ehime University \\ 3 Bunkyo-cho, Matsuyama, Ehime 790-8577, Japan \\ E-mail: iwamoto.yukiharu.mc@ehime-u.ac.jp \\ *** Tadano Ltd. \\ 5405-3 Shido, Sanuki, Kagawa 769-2101, Japan \\ $\dagger$ Caterpillar Japan Ltd. \\ 1106-4 Shimizu, Uozumi-cho, Akashi, Hyogo 674-8686, Japan \\ $\dagger$ Japan Atomic Energy Agency \\ 4002 Narita-cho, O-arai-machi, Ibaraki 311-1393, Japan
}

\begin{abstract}
Laser Doppler Velocimetry (LDV) measurements in a 90 degree elbow of which the curvature radius coincides with its inner diameter were examined for the cases of inflow from a long pipe, short pipe and swirl generator. Ensemble averaged flow distribution at the Reynolds number of 320000 based on the inner pipe diameter and bulk velocity shows that shortening the upstream pipe length to $4.9 \mathrm{D}$ from $10 \mathrm{D}$ induces the flow separation downstream of the elbow. Detailed observation suggests that shortening upstream pipe weakens the Prandtl's secondary flow of the first kind. Our swirl generator induced a swirling inflow with the non-dimensional angular momentum of 0.12 based on the inner pipe diameter and bulk velocity. The circumferential velocity distribution formed a shape like a Rankine combined vortex at the elbow inlet, and the accelerated axial velocity was observed at the vortex center. The axial velocity distribution however was found to be almost the same as that of the non-swirl inflow case in the latter half of the elbow. Frequency analyses showed that the Strouhal number by vortex shedding from the boundary layer occurring at the inner side of the elbow become 0.5 , except for 0.6 in the case of the long pipe. The change of the Strouhal number is probably related with the boundary layer width and the local flow velocity.
\end{abstract}

Key words : Elbow, Flow Induced Vibration, Developed Inflow, Undeveloped Inflow, Swirling Inflow, LDV

\section{Introduction}

Curved pipes/ducts are essential piping elements and widely used in heat exchangers, chemical plants and waterworks. It is well known that the pressure loss generated in a curved pipe differs from that in a straight pipe since the flow in the former one forms peculiar twin longitudinal vortices referred as the secondary flow of Prandtl's first kind. ${ }^{(1)}$ Therefore investigations of a flow in a curved pipe, especially an elbow, which is frequently used in an industrial field, have commercial and academic significance. Many kinds of experimental and theoretical studies of them have been done; e.g. yielding the formula to predict the pressure loss, ${ }^{(2),(3)}$ an improvement of efficiency by a guide vane, ${ }^{(4)}$ an analytical survey showing that the velocity gradient normal to the curvature plane generates the streamwise vorticity, ${ }^{(5)} \mathrm{nu}$ merical investigation and physical interpretation of multiple solutions in curved rectangular ducts, ${ }^{(6),(7)}$ numerical validations for turbulence models. ${ }^{\left({ }^{(8)},(9)\right.}$

*Received 4 June, 2012 (No. 12-0244) [DOI: 10.1299/jfst.7.315]

Copyright (c) 2012 by JSME 
Needless to say, a flow-induced vibration should be suppressed or at least avoided for a safety operation of piping systems. Recently, several researchers have pointed out that a flow in an elbow has the unique flow-induced vibration that has the Strouhal number (based on the bulk velocity and inner pipe diameter) of 0.5 occurring in the elbow downstream region. ${ }^{(10)-(12)}$ We have investigated this vibration precisely by LDV measurements and flow visualizations, and found that it is caused by the alterative vortex shedding form the circumferential edges (in the cross section of the pipe) of the boundary layer occurring at the elbow inside. ${ }^{(13)-(15)}$ Moreover its Strouhal number is consistent with that of Karman vortex when estimated by the local flow velocity convecting vortices and the circumferential width of the boundary layer, i.e. distance between alternative vortices.

The velocity profile in a bend depends on an inflow condition. Taylor et al. ${ }^{(16)}$ have made LDV measurements for a curved duct with curvature/hydraulic diameter of 2.3 and the Reynolds number of 60000 . Their duct was identical to that of Humphrey, ${ }^{(17)}$ but the upstream tangent was shortened from 45 to 7.5 times of the hydraulic diameter. They mentioned that the difference was apparent over the first 60 degrees of the bend due to the secondary flow. The flow variation by changing the length of the upstream tangent has been also pointed out in a hotwire anemometry for a curved circular pipe by Sudo and Hibara ${ }^{(18)}$ under $R_{c} / D=7.5$ and $R_{e}=120000$. Swirling inflow is also the cause deforming a flow profile. It is interesting that the weak swirling inflow occasionally reduces a friction in a curved pipe. ${ }^{(19)}$ These inflow conditions are not special cases but commonly encountered in actual piping systems. The present study has been started in order to obtain the data in hot leg piping for a safety operation of Japan sodium-cooled fast reactor (JSFR), that has been planned by Japan Atomic Energy Agency (JAEA). It is important to extend our knowledge of inflow effects to various cases, especially on the flow-induced vibration, since various inflows are expected at the hot leg inlet considering an arrangement of components in the reactor.

This paper shows the results of LDV measurements in an elbow with $R_{c} / D=1$ using a long pipe, a short pipe connected to a buffer tank, and a swirl generator as the upstream piping element. Details of the experiment are mentioned in Section 3, and results for each case relating with the other cases are shown in Section 4 . Section 4 also compares our result with that of the $1 / 3$ scale hot leg piping model of JSFR examined by Shiraishi et al. ${ }^{(11)}$ Finally, concluding remarks are made in Section 5.

\section{Nomenclature}

$D$ : inner diameter of the test piping

$f:$ frequency

$r:$ radial coordinate in a cross section

$R_{c}$ : curvature of the elbow

$R_{e}$ : Reynolds number, $\rho U_{m} D / \mu$

$S_{t}:$ Strouhal number, $f D / U_{m}$

$u$ : axial (streamwise) velocity

$v:$ normal (to the plane of curvature) velocity

Greek letters

$\theta:$ angle from an entrance of the elbow

$\mu$ : viscosity

Subscripts

$P S D$ : power spectrum density

$U_{m}$ : bulk velocity

$x$ : axial coordinate from an exit of the elbow

$x^{\prime}$ : axial coordinate from an entrance of the elbow

$z$ : coordinate in the direction of the curvature radius (positive to outward)

$$
\rho: \text { density }
$$

Superscripts

' : fluctuation component

$r m s$ : root mean square value

- : ensemble average

\section{Experimental Method}

\subsection{Overview of an Experimental Apparatus}

The experimental apparatus is schematically shown in Fig. 1. The experimental setup 
consists of a back pressure tank (volume: $1480 \times 980 \times 800 \mathrm{~mm}^{3}$, shown by (1) in Fig. 1), pump (Ebara 150×125FSW4H615, (2)), electro-magnetic flowmeter (Yokogawa AXF065GNNAL1S-BJ11-0NA, AXFA11P-D101, (3), settling chamber (4)), buffer tank (5), short pipe (6) and test section elbow (7)). The test section elbow is considered as a $1 / 3$ scale model of hot leg piping in JSFR. Tap water with a temperature between 18 and $35{ }^{\circ} \mathrm{C}$ was used as a working fluid. The inner diameter of the test section was $125 \mathrm{~mm}$ that coincided with the curvature of the center axis. The settling chamber was made with a PVC pipe with an inner diameter of $194 \mathrm{~mm}$ and was stuffed with a bundle of straws with a diameter of $13 \mathrm{~mm}$ and a length of $194 \mathrm{~mm}$. The other piping was almost made with a PVC and steel pipe except for a LDV measurement section that was made with an acrylic resin. In order to avoid the resonance between the structure and a flow-induced vibration, the setup was supported by stainless steel angles and fixed to a floor by anchor bolts. The height of the test section center from the floor was $930 \mathrm{~mm}$. A tapping test precedently examined had shown that the resonant frequency of the whole setup was $36 \mathrm{~Hz}$. In order to prevent the vibration from the pump, the pump was mounted on a vibration absorber (Tokkyokiki PX-130Z) and flexible joints (Zenshin ZRJ-B) were further installed between the pump and the piping.

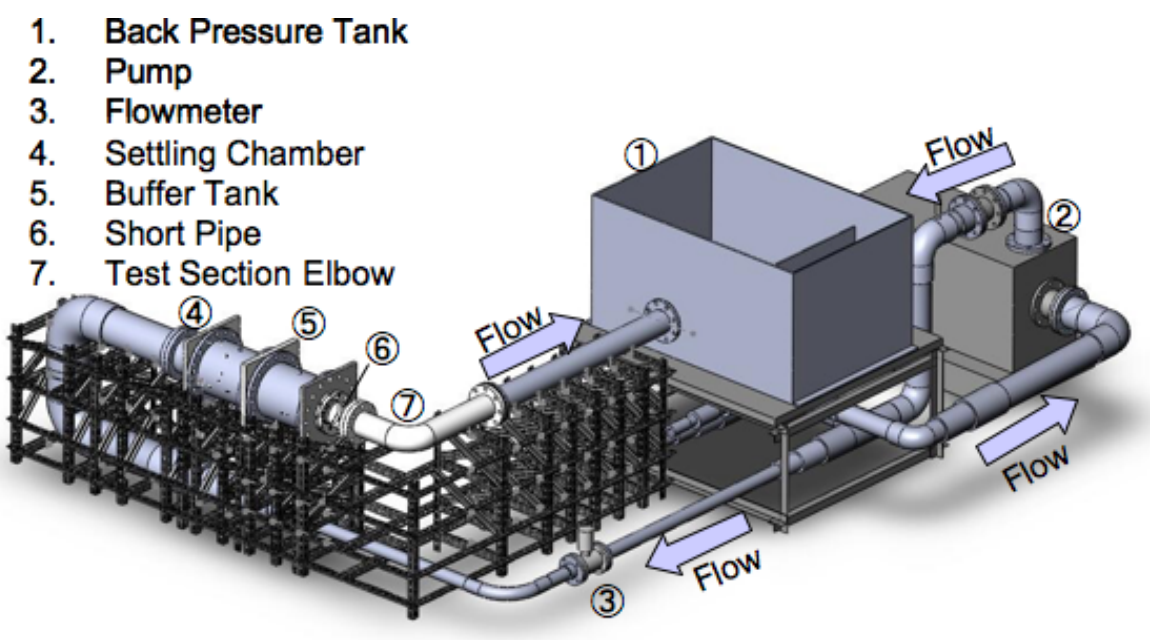

Fig. 1 Experimental apparatus (short pipe installed)

With intent to examine different inflow conditions, we also used a long pipe and swirl generator as an alternative of the combination of the short pipe and buffer tank. Each of these equipments has the following purposes: Results of long and short pipes inform us the effect of the length of the upstream tangent. The long/short pipe located upstream of the elbow will make a flow with a thick/thin boundary layer. The test with the swirl generator was also examined since the swirling inflow had a possibility to modify the vortex shedding that was mentioned in Section 1. Details of these upstream piping elements will be stated in the following subsections.

\subsection{Configuration of Long Pipe Case}

A contraction nozzle was set at the upstream side of the long pipe in order to remove effects from upstream piping elements. The contraction nozzle was made with an aluminum alloy (A2017), and its inner radius $R$ was determined by the third order polynomial ${ }^{(20)}$ as follows:

$$
R=\frac{D_{\text {in }}}{2}-\frac{3}{2}\left(D_{\text {in }}-D_{\text {out }}\right)\left(\frac{x}{L}\right)^{2}+\left(D_{\text {in }}-D_{\text {out }}\right)\left(\frac{x}{L}\right)^{3}
$$

Here $x$ is an axial coordinate from the nozzle entrance, $D_{\text {in }}$ and $D_{\text {out }}$ are respectively inner diameters at the nozzle entrance and exit (194 and $125 \mathrm{~mm}$ ). $L=100[\mathrm{~mm}]$ is the axial length of the nozzle. The pipe having an axial length of $10 D$ was put between the contraction nozzle 
and the test section elbow. In the present study, we refer this pipe as the "long pipe" in order to distinguish the short pipe that will be mentioned later. The downstream tangent between the exit of the elbow and back pressure tank measured $17 D$. The Reynolds number based on the inner diameter was set to 320000 (bulk velocity $U_{m} \approx 2[\mathrm{~m} / \mathrm{s}]$ ).

\subsection{Configuration of Short Pipe Case}

The upstream side of the short pipe was connected to the buffer tank. The buffer tank measured $286 \mathrm{~mm}$ in an inner diameter and $847 \mathrm{~mm}$ in an axial length, and was made with PVC. The buffer tank could be divided into two sections, and a baffle plate with the thickness of $35 \mathrm{~mm}$ was inserted between them for the purpose of canceling a flow development in the upstream region. The baffle plate was made with an aluminum alloy (A2017), and its shape was referred to Akashi et al. ${ }^{(21)}$ A bellmouth with an inlet/outlet diameter ratio of 1.15 was put into the end of the buffer tank, and the flow was introduced through the bellmouth into the short pipe, which the elbow followed. The axial length between the entrance of the bellmouth and the entrance of the elbow was $4.9 D$ that was about half of the long pipe case. The length between the exit of the elbow and the back pressure tank was the same as the long pipe case. The Reynolds number was adjusted to $320000\left(U_{m} \approx 2[\mathrm{~m} / \mathrm{s}]\right)$.

\subsection{Configuration of Swirl Generator Case}

Figure 2 shows a transparent view of the swirl generator used in the present study. The axial length was the same as the latter part of the buffer tank used in the short pipe case. A swirling inflow was introduced by replacing the latter part of the buffer tank with the swirl generator. 24 guide vanes made with A2017 were placed in an annular space between with inner and outer PVC pipes. Diameters of these pipes were respectively 216 and $286 \mathrm{~mm}$. Each of the guide vanes had the axial length of $69 \mathrm{~mm}$ and the height of $35 \mathrm{~mm}$. Their leading edges were hinged and circumferential displacements of their trailing edges achieved the swirling inflow. In the present study, guide vanes were uniformly tilted by 10 degrees against the axial direction. The axial length between the exit of the swirl generator and the entrance of the elbow was 3.6D. The swirl inflow experiment was done after rearrangement of the apparatus, so the length between the exit of the elbow and the back pressure tank was changed to $28 D$. The Reynolds number was kept with $407000\left(U_{m} \approx 2.7[\mathrm{~m} / \mathrm{s}]\right)$.

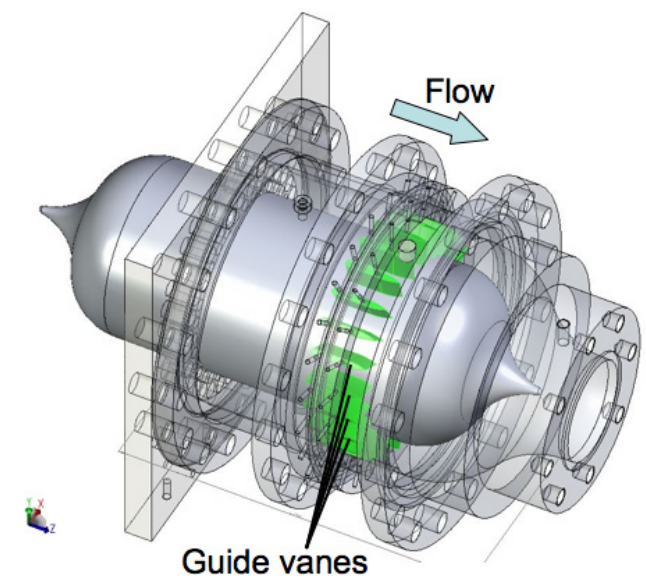

Fig. 2 Transparent view of the swirl generator

\subsection{LDV Measurement Technique}

Here we describe the details of the LDV measurement. The laser light source of the LDV was 6W argon-ion laser (Spectra physics Stabilite 2017), and the post-processor was Dantec 60X series. Green light (wave length: $514.5 \mathrm{~nm}$ ) was used for measuring an axial (streamwise) velocity. Blue light (wave length: $488 \mathrm{~nm}$ ) was also used when measuring a normal (to 
the plane including the curvature) velocity. Measuring volumes for axial and normal velocities respectively had the lengths of $48 / 45 \mu \mathrm{m}$ in measuring directions. Each light from the source was split into two laser beams. The intersection angle between these two laser beams changed because of refraction at borders between different materials. This was compensated as long as possible by a water jacket covering the elbow. A complete compensation could be done when the refraction rate of the elbow coincided to that of water, but not in the present study. This incomplete compensation posed errors in a fringe interval (proportional to the velocity) at the measuring point and a location of the measuring point. Errors of fringe intervals were respectively estimated at $0.2 / 1 \%$ in axial/normal velocities. Errors of measuring locations were estimated at $0.4 / 0.5 \%$ in axial/normal velocity measurements. Uncertainties of $\bar{u} / U_{m}, \bar{v} / U_{m}$ shown here are absolutely less than 0.04 according to ANSI/ASME PTC19.1-1985, ${ }^{(22)}$ including the errors in fringe intervals. The present LDV system adopted a back-scattered type, so the refraction and the attenuation of the scattered light easily lessened the measurement quality. To overcome this, a retroreflector mirror (Kanomax model 1807) was placed in the opposite side of the probe and the scattered burst light passing through the elbow was also utilized. A nylon powder with an average diameter of $4.1 \mu \mathrm{m}$ and specific gravity of 1.02 was used as seeding.

Sampling number of instantaneous velocities in each LDV measurements was determined to at least 60000 in order to saturate the statistical values, i.e. an ensemble average and turbulent intensity (a root mean square value of the velocity fluctuation). The data that deviates more than five times of the standard deviation form the average were disposed when calculating these statistical values. We also made Fast Fourier Transform (FFT) analyses for the velocity data. Prior to FFT analysis, the velocity data were modified by the third order spline interpolation into the 8 sets of data that had the same interval of 0.01 seconds and 4096 data points. Power spectrum density functions were calculated from these 8 sets of spline data, and ensemble averaging over these power spectrums was done to obtain the result removing an incidental error. All the LDV data used for FFT analyses had mean sampling rates of more than $80 \mathrm{~Hz}$ to keep the accuracy of spline data. Both the temperature (between 18 and 35 ${ }^{\circ} \mathrm{C}$ ) and the flow rate of the working fluid were always monitored and the constant Reynolds number was kept by adjusting the output of the inverter (Fuji electric FRN15F1S-2J) which was connected to the pump.

\section{Results and Discussion}

\subsection{Long Pipe Case}

Figure 3 shows axial velocity distributions using the long pipe (axial length: $10 D$ ) as the upstream piping element. Here $x^{\prime}$ is the axial coordinate measured from the elbow entrance, $\theta$ is the angle measured form the entrance of the elbow, and $x$ is the axial coordinate measured form the elbow exit. $z / D=-0.5$ and +0.5 respectively correspond to the inside and the outside of the curvature. The data in Fig. 3 have been taken under the Reynolds number of 320000 .

We hereafter mention how the velocity profile alters as the flow goes downstream. At $x^{\prime} / D=-9$ (corresponds to $1 D$ downstream from the contraction nozzle), averaged velocity becomes uniform except for regions adjacent to walls, indicating that the contraction nozzle effectively rectifies the flow. The turbulence intensity in the uniform velocity region is approximately 0.03 . The flow accelerates in the inside region at $x^{\prime} / D=-0.1$ in Fig. 3(a). The locally small pressure at the inside of the elbow causes this acceleration. The inside flow acceleration becomes obvious at $\theta=22.5^{\circ}, 45^{\circ}$ and $67.5^{\circ}$. At the same time, the turbulence intensity increases outside of the elbow, as shown by Fig. 3(b). This is because the flow near the outside wall is intrinsically unstable due to the unbalance between the inertia and the centrifugal force. ${ }^{(23)}$ Pressure gradients in the axial direction become adverse and favorable in the inside and outside regions, respectively, in the latter part of the elbow. Therefore the flow velocity in the downstream region $(x / D>0)$ becomes slower and faster in corresponding 




(a) Ensemble averaged velocity

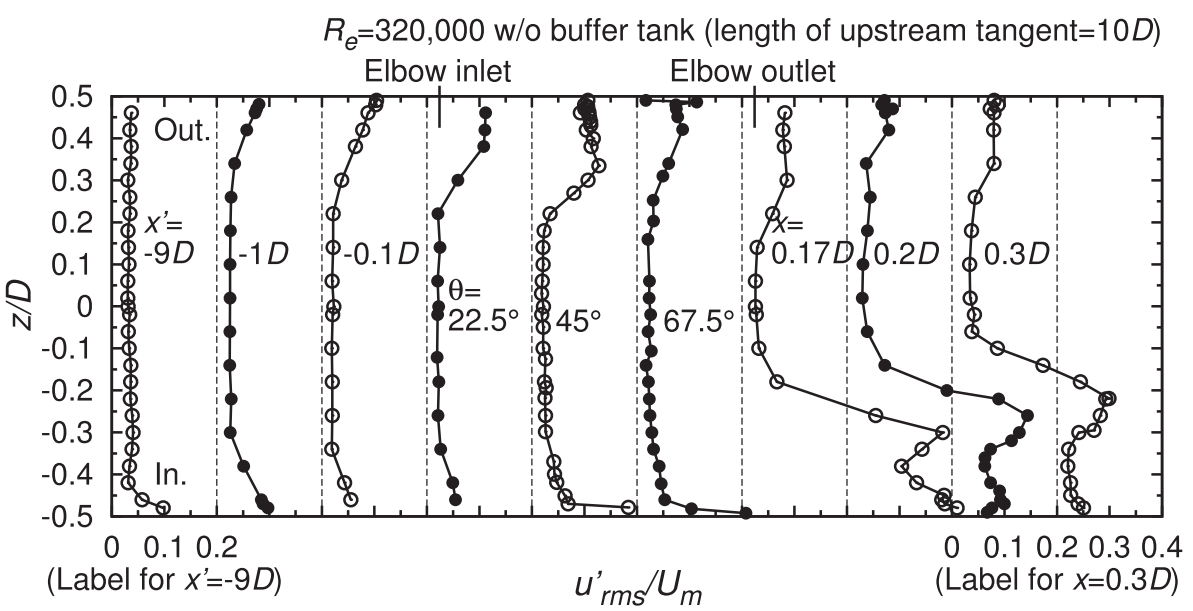

(b) Turbulent intensity

Fig. 3 Distributions of axial velocities (Long pipe case, $R_{e}=320000$ )

regions, forming the shear region as shown in $-0.35<z / D<-0.1$ in Fig. 3(a). This shear makes the turbulence intensity strong as shown in Fig. 3(b). As seen in $-0.5<z / D<-0.3$ at $x / D=0.3$ in Fig. 3(a), the averaged velocity distribution becomes convex near the inside of the elbow. This locally fast velocity originates from the fluid that is transported from the outside of the elbow by the secondary flow of Prandtl's first kind. The locally convex velocity profile has been also observed in a hotwire anemometry with $R_{e}=60000$ and $R_{c} / D=2$ by Sudo et al. ${ }^{(24)}$ and in a LDV measurement with $320000<R_{e}<3700000$ and $R_{c} / D=1.026$ by Shiraishi et al. (11) $^{\text {. }}$

Our previous study, which has been done under the same experimental condition except for the Reynolds number of 50000, has shown that the averaged flow separates in the region between $\theta=45^{\circ}$ and $x / D=0.3 .^{(13)-(15)}$ On the other hand, Fig. 3(a), with $R_{e}=320000$, shows that the averaged flow does not separate except for the only one point near the inside wall $(x / D=0.2, z / D=-0.48)$. One of the reasons for the difference whether the flow separates or not is the difference of inertia: The flow with $R_{e}=320000$ has larger inertia that endures the adverse pressure gradient. But this explanation is not sufficient since the pressure gradient also becomes strong in a larger Reynolds number case. It should be noted that results shown in Fig. 3(a) do not conclude that the flow does never separate. We can see that unsteady flow separation occurs near the inside wall in the downstream region since the turbulence intensities are sufficiently large in the corresponding area.

Figure 4 shows power spectrums of axial velocitiy fluctuations for points in the outer region (a), the flow shear region (b) and the inner region (c) at $x / D=0.17$. Fluctuation with the Strouhal number of $0.6(10.3 \mathrm{~Hz})$ are found in shear and inside regions, as are seen 


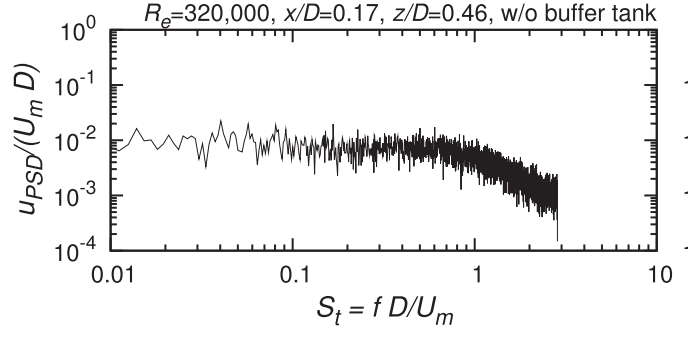

(a) $z / D=+0.46$

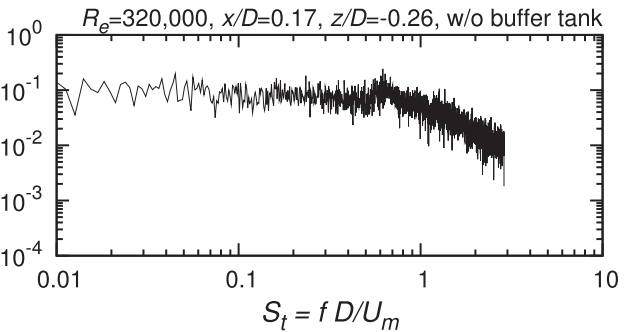

(b) $z / D=-0.26$

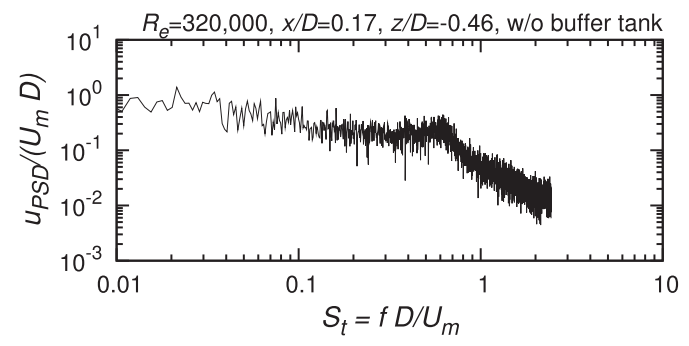

(c) $z / D=-0.46$

Fig. 4 Power spectrums of axial velocity fluctuations (Long pipe case, $R_{e}=320000$ )

in Figs. 4(b) and (c). Observation of the other region reveals that this fluctuation can be found in the inside region including the flow shear region downstream of the elbow, not in the elbow. Our previous study with $R_{e}=50000^{(13)-(15)}$ has been clarified that this dominant fluctuation corresponds to the alternative vortex shedding from the circumferential edges (in the cross section of the pipe) of the boundary layer occurring inside of the elbow. However two differences exist between the previous and present study: (1) the Strouhal number becomes faster than previous one $\left(S_{t}=0.5\right)$, and (2) no steady flow separation occurs in the present study. The item (2) shows that this periodic vortex shedding can occur even if the shear is not so strong that the flow separates.

\subsection{Short Pipe Case}

Figure 5 shows axial velocity profiles in the case using the short pipe (axial length: 4.9D). Axes in the figure and the operating Reynolds number are the same as in Fig. 3. The steady flow separation near the inside of the curvature $(z / D<-0.35)$ can be found in the downstream locations $(x / D=0.17,0.2$ and 0.3$)$. This is the most distinct difference between the long and short upstream pipe cases. The velocity distribution at $x^{\prime} / D=-1$ in the case of the long pipe is overwritten by open square red symbols in Fig. 5(a). (denoted by "w/o buffer tank") We can see that the velocity distribution of the short pipe case, denoted by closed circle symbols, becomes flat compared to the long pipe case. This means that the total inflow axial momentum becomes smaller in the short pipe case, suggesting that the pressure gradient along with the curvature radius, which is necessary to turn the flow, becomes small in the short pipe case. This smaller pressure gradient induces smaller secondary flow, so the transport of the fluid with faster velocity from the outside becomes weaken. It is possible that the flow separation is caused by the lack of the secondary flow. The defect of the secondary flow is also supported by the fact that the locally convex velocity distribution near the inside wall becomes alleviated. Taylor et al. ${ }^{(16)}$ have made LDV measurements for a square curved duct and pointed out that the secondary flow becomes strong in the case of the smaller core inflow. This situation also applies to the present case.

Figure 6 shows power spectrums of axial velocity fluctuations at $x / D=0.17$ shown in Fig. 5. No remarkable fluctuation is found in the elbow as is in the long pipe case. Fluctuation with the Strouhal number of $0.5(8.6 \mathrm{~Hz})$ is found only in the inside region as seen in Fig. 6(c). However the corresponding frequency peak is blunt. One possibility for the blunt peak is that the center of the shedding vortex, i.e. source of the fluctuation, becomes apart from the 


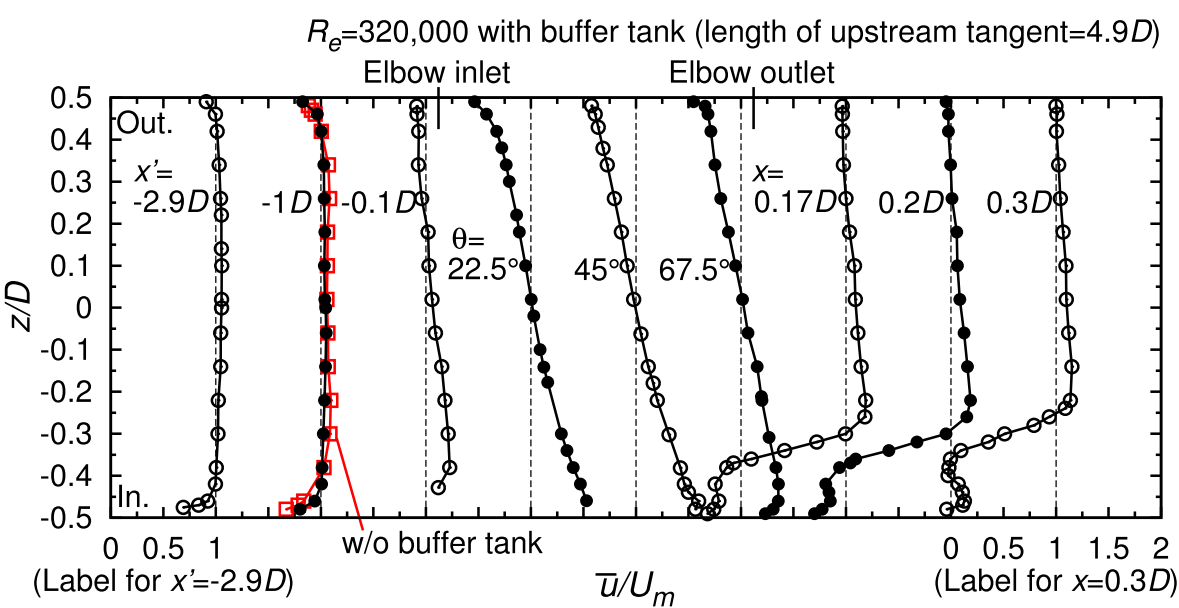

(a) Ensemble averaged velocity

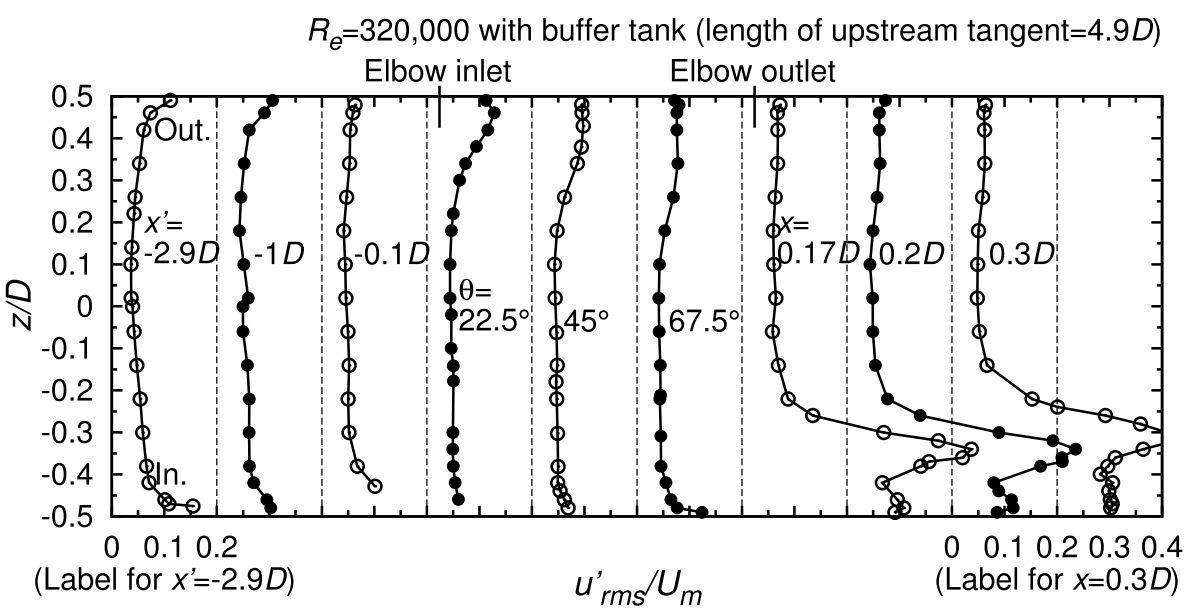

(b) Turbulent intensity

Fig. 5 Distributions of axial velocities (Short pipe case, $R_{e}=320000$ )

measuring point (center of the pipe). Figure 7 shows the power spectrum at the location $x / D=$ $0.17, r / D=0.484$ and 10 degrees apart from the elbow inside. The fluctuation attenuates while the measuring point moves from the vortex core (near the point in Fig. 7) to the center plane (Fig. 6(c)).

\subsection{Swirl Generator Case}

Figures 8 and 9 respectively show axial and normal velocity distributions in the case of the swirl generator. The Reynolds number was kept to be 407000 . At the most upstream measurement location, $x^{\prime} / D=-2.9$, a symmetric swirling inflow like a Rankine combined vortex is seen as shown by Figs. 8(a) and 9(a). The non-dimensional angular momentum

$$
\Omega=\frac{16}{D^{3} U_{m}^{2}} \int_{0}^{D / 2} r^{2} \bar{u} \bar{v} d r
$$

at $x^{\prime} / D=-2.9$ is calculated to be 0.12 . The axial velocity and its root mean square value become large at the pipe center in the upstream region. This indicates that the pressure at the pipe center becomes locally small and the vortex center is fluctuating around the pipe center. We observed small cavitations meandering there, but it does not affect the result because the results obtained here do not have a drastic change compared to the other results shown here.

Comparison of Figs. 5(a) and 8(a) provides that the difference between them is small in the elbow and its downstream region, except that the velocity profiles at $\theta=22.5^{\circ}$ and $45^{\circ}$ in Fig. 8(a) have folds near $z / D=-0.25$. This is caused by the convection of the swirling flow. Sudo et $a l .{ }^{(25)}$ also have shown the deformation by the swirling inflow under the condition of 


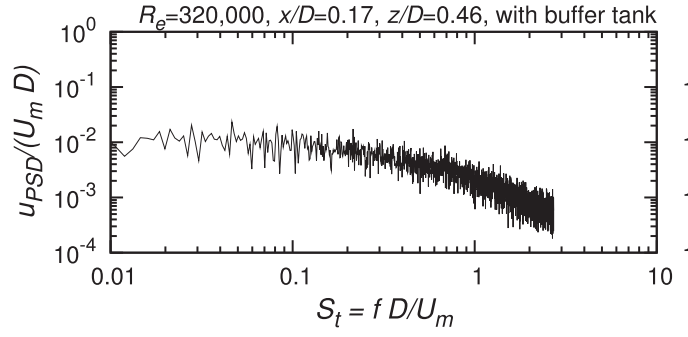

(a) $z / D=+0.46$



(b) $z / D=-0.36$



(c) $z / D=-0.49$

Fig. 6 Power spectrums of axial velocity fluctuations (Short pipe case, $R_{e}=320000$ )

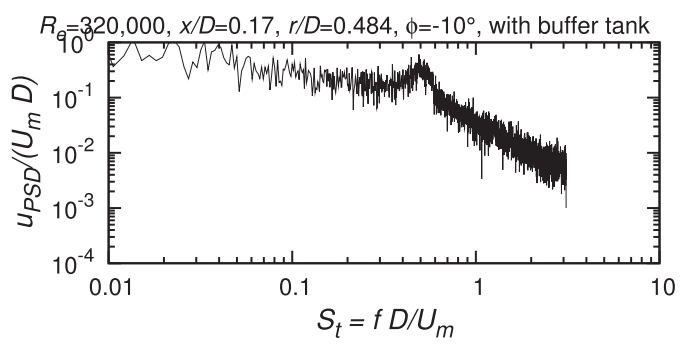

Fig. 7 Power spectrum of the axial velocity fluctuation at $x / D=0.17$ and $r / D=0.484$, $10^{\circ}$ apart from the elbow inside (Short pipe case, $R_{e}=320000$ )

$R_{e}=60000, R_{c} / D=2$ and non-dimensional angular momentum of 0.075 , that is much less compared to our study.

We can consider two reasons for the small difference between Figs. 5(a) and 8(a). One reason is that the attenuation of the swirling flow is promoted by an interaction with a secondary flow in a bend. Sudo and Takami ${ }^{(26)}$ have shown that the non-dimensional angular momentum changed from 0.57 and 0.32 to 0.28 and 0.15 respectively while passing through the bend. They explain that the attenuated swirl flow has the distribution that the large axial velocity region has the small swirl velocity and vice versa. Our case have the non-dimensional angular momentum 0.12, much less than Sudo and Takami, so attenuated swirl flow hardly have the circumferential velocity. The other reason is that the swirling vortex core moves far from the measuring plane and the induced velocity by the vortex becomes very weak in Fig. $8(\mathrm{a})$.

Figure 10 shows power spectrums of the axial velocity fluctuations at $x / D=0.17$ shown in Fig. 8. No remarkable fluctuation is found in the elbow as are in two cases mentioned above. The fluctuation with the Strouhal number of $0.5(10.4 \mathrm{~Hz})$ is found both in the velocity shear and the inside in the downstream region, as seen in Figs. 10(b) and (c). The spectrum peak is sharp, comparing with Figs. 4 and 6. The swirling inflow may restrict the scattering of vortices shedding from the boundary layer occurring inside of the elbow.

\subsection{Summary of All Cases}

Table 1 summarizes the results of past ${ }^{(13)-(15)}$ and present studies in order to clarify the effect of the inflow in the elbow, especially on the remarkable frequency corresponding to the alternative vortex shedding from the inside boundary layer. In case (A) in Table 1, that 




(a) Ensemble averaged velocity

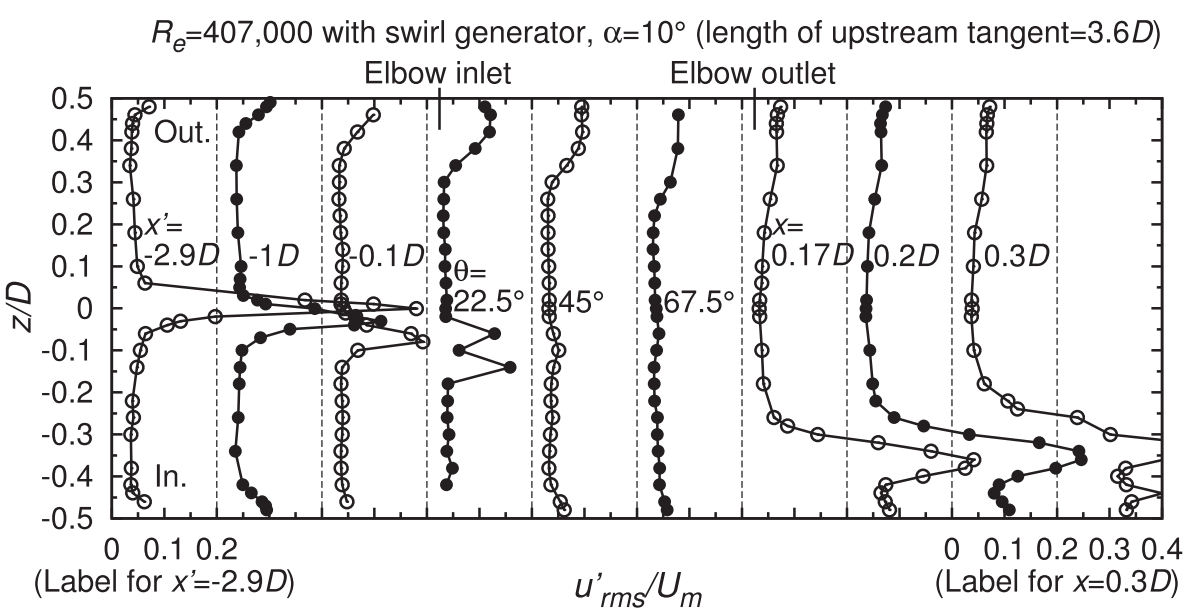

(b) Turbulent intensity

Fig. 8 Distributions of axial velocities (Swirl generator case, $R_{e}=407000$ )

has been previously done, two remarkable Strouhal numbers are found, but not in others. Flow visualization has shown that the higher Strouhal number in case (A) seems to be the result of detecting the fluctuation from the two alternative shedding vortices. The difference between case (A) and the others are Reynolds numbers. The dissipation will be strong in larger Reynolds number cases, so the higher frequency found in case (A) will disappear.

The Strouhal number increases to 0.6 only in case (B). The obvious difference between case (B) and the others is that the flow does not separate in the steady meaning while the others separate. This difference leads two possibilities for increasing the Strouhal number of the vortex shedding, assuming that there are the proper velocity and length to obtain the universally constant Strouhal number: One is the shrink of the boundary layer. This will decrease the distance between the vortices. The other is the flow velocity, which convects vortices, increases in the case of no flow separation. Anyway the Strouhal number is sensitive to the flow distribution in the downstream region.

\subsection{Comparison with $1 / 3$ Scale Model}

Shiraishi et al. ${ }^{(11)}$ have made LDV measurements for an elbow that has the inner diameter of $412.7 \mathrm{~mm}$ and the curvature/diameter ratio of 1.026 . Their elbow was utilized as the $1 / 3$ scale model of JSFR. On the other hand, our elbow applies the inner diameter of $125 \mathrm{~mm}$ and almost the same curvature/diameter ratio, i.e. 1.0, and is regarded as the geometrically similar shape with the $1 / 3$ scale model. So comparison with their data can validate our data. Figure 11 shows the result. The closed circle and open triangle indicate the results of the long pipe cases. The boundary layer becomes thinner and the profile approaches to the data of Shiraishi 




(a) Ensemble averaged velocity

$R_{e}=407,000$ with swirl generator, $\alpha=10^{\circ}$ (length of upstream tangent $=3.6 D$ )

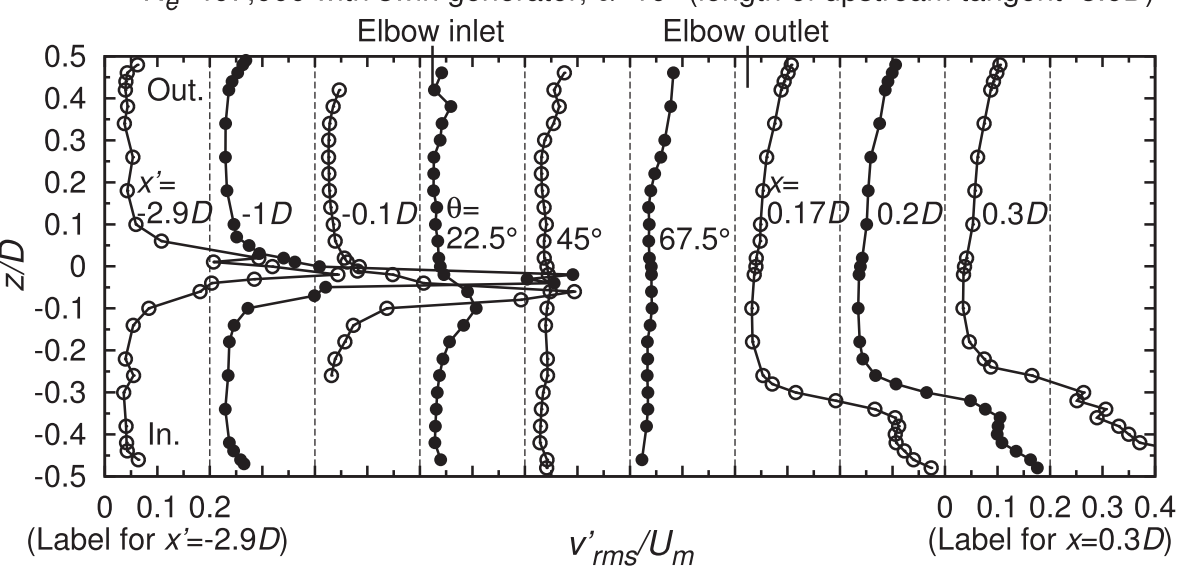

(b) Turbulent intensity

Fig. 9 Distributions of normal velocities (Swirl generator case, $R_{e}=407000$ )

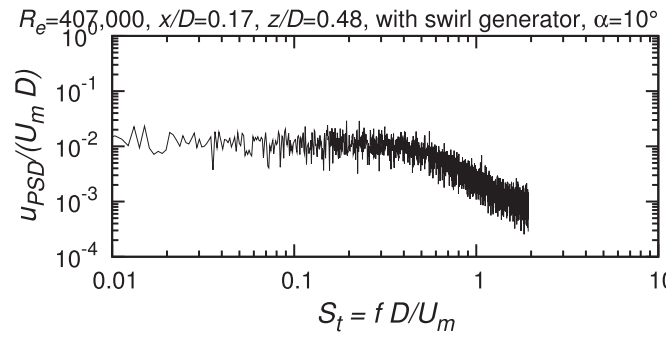

(a) $z / D=+0.48$



(b) $z / D=-0.36$

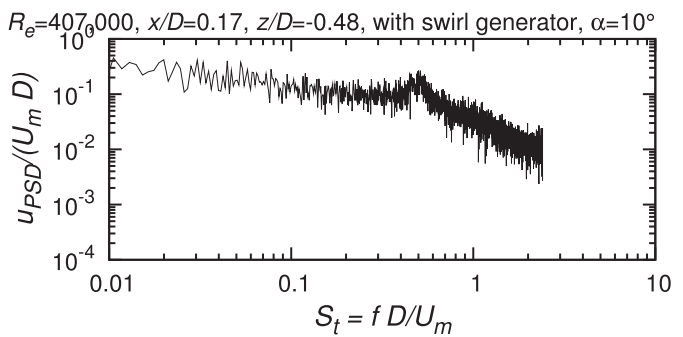

(c) $z / D=-0.48$

Fig. 10 Power spectrums of axial velocity fluctuations (Swirl generator case, $R_{e}=$ 407000)

et al. as the Reynolds number increases. The flow profile further approaches when using the short pipe, shown by the closed triangle in Fig. 11. The short pipe measures $4.9 D$ that is close to the case of Shiraishi et al. (5.6D) Figure 11 indicates that the flow profile downstream of 
Table 1 Summary of past ${ }^{(13)-(15)}$ and present LDV measurements

\begin{tabular}{|c|c|l|}
\hline Inflow condition & Strouhal number & \multicolumn{1}{|c|}{ Remark } \\
\hline $\begin{array}{c}\text { (A) From long pipe (axial length: } \\
10 D), R_{e}=50000^{(13)-(15)}\end{array}$ & $\begin{array}{l}1.0(3.2 \mathrm{~Hz}), \\
0.5(1.6 \mathrm{~Hz})\end{array}$ & $\begin{array}{l}S_{t}=1.0 \text { in the shear region, } \\
S_{t}=0.5 \text { in the boundary layer } \\
\text { at the elbow inside }\end{array}$ \\
\hline $\begin{array}{l}\text { (B) From long pipe (axial length: } \\
10 D), R_{e}=320000\end{array}$ & $0.6(10.3 \mathrm{~Hz})$ & $\begin{array}{l}\text { Occurs both in the shear region } \\
\text { and boundary layer at the elbow } \\
\text { inside }\end{array}$ \\
\hline $\begin{array}{l}\text { (C) From short pipe (axial length: } \\
4.9 D), R_{e}=320000\end{array}$ & $0.5(8.6 \mathrm{~Hz})$ & $\begin{array}{l}\text { Occurs both in the boundary } \\
\text { layer at the elbow inside, weak } \\
\text { spectrum peak }\end{array}$ \\
\hline $\begin{array}{l}\text { (D) From swirl generator (non- } \\
\text { dimensional angular velocity: } \\
0.12, \text { upstream pipe length: } \\
3.6 D), R_{e}=407000\end{array}$ & $0.5(10.4 \mathrm{~Hz})$ & $\begin{array}{l}\text { Occurs both in the shear region } \\
\text { and boundary layer at the elbow } \\
\text { inside, distinct spectrum peak }\end{array}$ \\
\hline
\end{tabular}

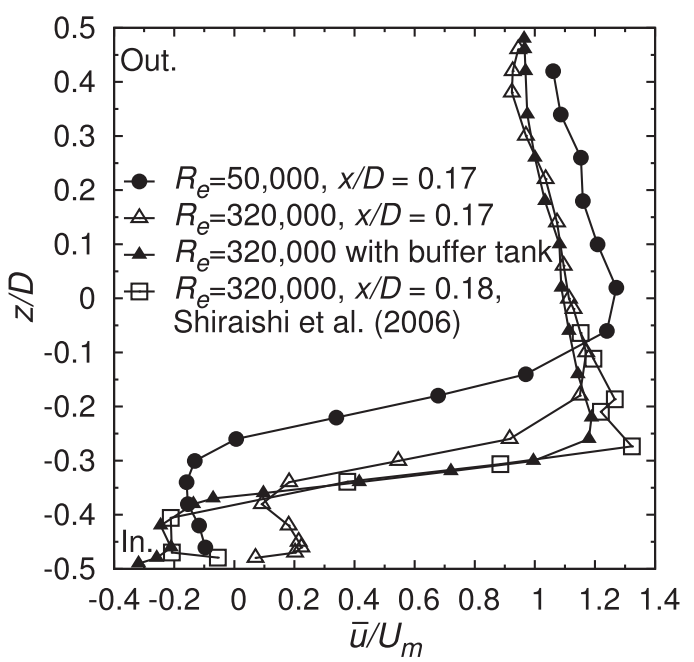

Fig. 11 Comparison of the axial velocities with the data of Shiraishi et al. ${ }^{(11)}$

the elbow is strongly influenced by the inflow condition.

\section{Conclusions}

LDV measurements in a 90 degree elbow have been examined for cases of inflow from a long pipe, short pipe and swirl generator. Conclusions are summarized as follows:

(1) The flow separates in the short pipe case, but not in the long pipe case at $R_{e}=$ 320000. This depends on the total momentum of the inflow that determines the strength of the secondary flow.

(2) The swirling inflow in the present case does not alter the flow profile in the elbow and its downstream region.

( 3 ) Strouhal numbers related with the vortex shedding from the inside boundary layer become 0.5 in the most of the cases, except for 0.6 in the long pipe case. The Strouhal number is sensitive to the flow distribution in the downstream region.

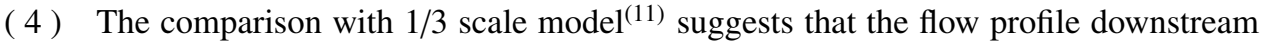
of the elbow is strongly influenced by the inflow condition.

\section{References}

( 1 ) Hawthorne, W. R., Secondary Circulation in Fluid Flow, Proceedings of the Royal Society of London, Series A, Vol. 206, No. 1086 (1951), pp. 374-387. 
( 2 ) Ito, H., Friction Factors for Turbulent Flow in Curved Pipes, Transactions of the ASME, Series D, Journal of Basic Engineering, Vol. 81, No. 2 (1959), pp. 123-134.

( 3 ) Ito, H., Pressure Losses in Smooth Pipe Bends, Transactions of the ASME, Series D, Journal of Basic Engineering, Vol. 82, No. 1 (1960), pp. 131-143.

( 4 ) Ito, H. and Imai, K., Pressure Losses in Vaned Elbows of a Circular Cross Section, Transactions of the ASME, Series D, Journal of Basic Engineering, Vol. 88, No. 3 (1966), pp. 684-685.

( 5 ) Squire, H. B. and Winter, K. G., The Secondary Flow in a Cascade of Airfoils in a Nonuniform Stream, Journal of Aeronautical Science, Vol. 18, No. 4 (1951), pp. 271277.

( 6 ) Yanase, S., Daikai, R. and Morinaga, T., Multiple Solution of a Flow in a Curved Rectangular Tube: 1st Report, Symmetric Steady Solutions and Their Stability, Transactions of the Japan Society of Mechanical Engineers, Series B, Vol. 64, No. 626 (1998), pp. 3183-3190.

( 7 ) Yanase, S., Daikai, R., Kaga, Y. and Yamamoto, K., Multiple Solution of a Flow in a Curved Rectangular Duct: 2nd Report, Resistance Coefficient of the Duct Flow, Transactions of the Japan Society of Mechanical Engineers, Series B, Vol. 65, No. 639 (1999), pp. 3622-3628.

( 8 ) Sugiyama, H., Hitomi, D., Numerical analysis of developing turbulent flow in a $180^{\circ}$ bend tube by an algebraic Reynolds stress model, International Journal for $\mathrm{Nu}$ merical Methods in Fluids, Vol. 47, No. 12 (2005), pp. 1431-1449.

( 9 ) Crawford, N. M., Cunningham, G. and Spence, S. W. T., An Experimental Investigation into the Pressure Drop for Turbulent Flow in $90^{\circ}$ Elbow Bends, Proceedings of the Institution of Mechanical Engineers, Part E: Journal of Process Mechanical Engineering, Vol. 221, No. 2 (2007), pp. 77-88.

(10) Kawamura, T. Nakao, T. and Takahashi, M., Reynolds Number Effect on Turbulence downstream from Elbow Pipe, Transactions of the Japan Society of Mechanical Engineers, Series B, Vol. 68, No. 667 (2002), pp. 645-651.

(11) Shiraishi, T., Watakabe, H., Sago, H., Konomura, M., Yamaguchi, A. and Fujii, T., Resistance and Fluctuating Pressures of a Large Elbow in High Reynolds Numbers, Transactions of the ASME, Journal of Fluids Engineering, Vol. 128, No. 5 (2006), pp. 1063-1073.

(12) Monji, H., Ojima, S. and Nakatani, T., Error Estimation of Ultrasonic Flowmeter Downstream of Elbow, Journal of the Japanese Society for Experimental Mechanics, Vol. 9, Special Issue (2009), pp. 71-75.

(13) Iwamoto, Y., Yasuda, K., Sogo, M., Yamano, H. and Kotake, S., Study on Flow-inducedvibration evaluation of Large-Diameter Pipings in a Sodium-Cooled Fast Reactor: (4) Experiments on the 1/10-scale Hot Leg Test Facility in Reynolds Number of 50000 and 320000, Proceedings of the Sixth Japan-Korea Symposium on Nuclear Thermal Hydraulics and Safety, (2008), N6P1010, 6 pp.

(14) Iwamoto, Y., Minamiura, H., Sogo, M. and Yamano, H., LDV Measurements of a Flow in a Strongly-Curved Elbow under a High Reynolds Number Condition, Transactions of the Japan Society of Mechanical Engineers, Series B, Vol. 75, No. 765 (2010), pp. 830-838.

(15) Iwamoto, Y., Sogo, M., Aizawa, K., Nakanishi, S. and Yamano, H., Basic Study on Flow Separation Phenomenon at the Elbow in a Large-Diameter Pipe; Research Report in FY2007, JAEA-Reserach 2010-009 (2010), 58 pp.

(16) Taylor, A. M. K. P., Whitelaw, J. M. and Yianneskis, M., Curved Ducts With Strong Secondary Flow Motion: Belocity Measurements of Developping Laminar and Turbulent Flow, Transactions of the ASME, Journal of Fluids Engineering, Vol. 104, No. 3 (1982), pp. 350-359.

(17) Humphrey, J. A. C., Whitelaw, J. H. and Yee, G., Turbulent flow in a square duct with 
strong curvature, Journal of Fluid Mechanics, Vol. 103 (1981), pp. 443-463.

(18) Sudo, K. and Hibara, H., Developing Turbulent Flow in a Curved Pipe, Transactions of the Japan Society of Mechanical Engineers, Series B, Vol. 65, No. 639 (1999), pp. 3629-3636.

(19) Takami, T., Yanase, S., Maruyama, Y. and Araki, K., Drag Reduction of Turbulent Flows in a Curved Pipe due to the Swirling Instability, Transactions of the Japan Society of Mechanical Engineers, Series B, Vol. 66, No. 644 (2000), pp. 997-1003.

(20) Hussain, A. K. M. F. and Ramjee, V., Effects of the Axisymmetric Contraction Shape on Incompressible Turbulent Flow, Transactions of the ASME, Journal of Fluids Engineering, Vol. 98, No. 1 (1976), pp. 58-69.

(21) Akashi, K., Watanabe, H. and Koga, K., Development of New Rectifier for Shortening Upstream Pipe Length of a Flowmeter, Proceedings of IMEKO Symposium on Flow Measurement and Control in Industry, (1979), pp. 279-284.

(22) American National Standard Institute and American Society of Mechanical Engineers, Measurement Uncertainty, (1985), ANSI/ASME PTC19.1-1985.

(23) Herbert, O. ed., Prandtl-Essentials of Fluid Mechanics, 3rd Ed., (2010), p. 309.

(24) Sudo, K., Sumida, M. and Hibara, H., Experimental Investigation on Turbulent Flow in a Circular-Sectioned 90-Degree Bend, Experiments in Fluids, Vol. 25, No. 1 (1998), pp. $42-49$.

(25) Sudo, K., Takami, H., Nomura, T. and Sumida, M., Weakly Swirling Flows through a $90^{\circ}$ Bend, Transactions of the Japan Society of Mechanical Engineers, Series B, Vol. 63, No. 606 (1997), pp. 505-512.

(26) Sudo, K. and Takami, T., Swirling Flows in a Circular-Sectioned $90^{\circ}$ Bend, Transactions of the Japan Society of Mechanical Engineers, Series B, Vol. 60, No. 570 (1994), pp. 464-472. 